\title{
Undersampling Correction for Array Detector-Based
}

\section{Satellite Spectrometers}

\author{
Kelly Chance, Thomas P. Kurosu, and Christopher E. Sioris \\ Harvard-Smithsonian Center for Astrophysics, 60 Garden Street, Cambridge, MA 02138, USA
}

Array detector-based instruments are now fundamental to measurements of ozone and other atmospheric trace gases from space in the ultraviolet, visible, and infrared. The present generation of such instruments suffers, to a greater or lesser degree, from undersampling of the spectra, leading to difficulties in the analysis of atmospheric radiances. We provide extended analysis of the undersampling suffered by modern satellite spectrometers, which include GOME, SCIAMACHY, OMI, and OMPS. The analysis includes basic undersampling, the effects of binning into separate detector pixels, and the application of high-resolution Fraunhofer spectral data to correct for undersampling in many useful cases.

(C) 2004 Optical Society of America

OCIS codes: $010.1280,120.4570,280.1120$

\section{Introduction}

Array-based spectrometers used in atmospheric remote sensing can suffer substantially from spectral undersampling, with negative consequences to the quality of data retrieved from the 
measurements. This was first obvious in measurements from the Global Ozone Monitoring Experiment (GOME), ${ }^{1}$ where scientists fitting trace gases from GOME spectra found quite large systematic fitting residuals, and large fitting errors, even after correcting for Doppler shifts between radiances and irradiances. Ref. 2 recognized that this was mainly due to spectral undersampling and presented a technique for correcting most (>90\%) of the undersampling error in spectral regions where atmospheric absorption effects are small. For GOME, this includes fitting regions used for nitrogen dioxide $\left(\mathrm{NO}_{2}\right)$, bromine monoxide $(\mathrm{BrO})$, chlorine dioxide (OClO), and formaldehyde (HCHO). If used with caution, the technique can also be applied successfully to $\mathrm{O}_{3}$ and $\mathrm{SO}_{2}$. (Caution is required because the absorption optical depths for $\mathrm{O}_{3}$ are higher than for the other trace species listed; correction assumes that, to first order, the radiance spectrum consists mostly of back scattered Fraunhofer structure. The $\mathrm{SO}_{2}$ absorption occurs in the region where $\mathrm{O}_{3}$ also absorbs strongly.) The technique consists of comparing fully-sampled and undersampled versions of a high resolution Fraunhofer reference spectrum, ${ }^{3}$ with the difference being the effect of undersampling. Ref. 4 suggested that the observed residuals are induced by the resampling required to compare Earth radiance and solar irradiance spectra in the fitting, because they are measured with different Doppler shifts of the ERS-2 satellite with respect to the sun. It is demonstrated here that this is not entirely the case: Wavelength shifts between GOME radiances and irradiances are larger than can be accounted for by Doppler shifts. Ref 4 also presented a version of the technique of Ref. 2 implemented in the Scanning Imaging Absorption Spectrometer for Atmospheric Chartography (SCIAMACHY) operational processor. The technique is now also used for measurement of $\mathrm{HCHO}^{5}$ and $\mathrm{NO}_{2}{ }^{6}$ and has been implemented in the operational processor for measurements of $\mathrm{BrO}, \mathrm{OClO}$, and $\mathrm{HCHO}$ by the Ozone Monitoring Instrument (OMI). ${ }^{7}$ 
The present analysis improves on the previous understanding of correction for undersampling by fully considering the sampling theorem in conjunction with the instrument slit function. The analysis is applied to measurements by GOME and OMI, since they represent the two major instrument types: diode array detectors (GOME) and CCD detectors (OMI). The development applies as well to other instruments, including SCIAMACHY (diode array detectors) and the Ozone Mapping and Profiler Suite (OMPS), part of the National Polar Orbiting Environmental Satellite System (NPOESS), which uses CCD detectors.

\section{Definitions and Assumptions}

The spectrometers considered here use array detectors, which respond to the incoming light over finite regions, with defined response profiles. We will use the term instrument line shape (ILS) for the response of a spectrometer to a monochromatic source up to where it enters the array detector, and the term instrument transfer function (ITF) for the ILS convolved with the detector pixel response. The following properties are assumed to be valid over the region of the array detector that is necessary to consider for ITFs and undersampling corrections at a given pixel, as developed here.

1. Linear response. Each pixel responds linearly to input light intensity.

2. Equal pixel response. Pixels respond equally to photons of different wavelength. Pixels respond (or can be calibrated to respond) equally in output signal for equal input light intensity.

3. Linear dispersion. Pixel wavelengths are at equal increments.

4. No endpoint issues. The region under consideration is sufficiently far from the array end for wavelengths beyond the array wavelength range to contribute to the signal. 
(Proximity to the array end would require re-calculation of the ILS from deconvolution of the ITF, as discussed below.)

5. Calibration light source issues. Instrument transfer functions (ITFs) are determined using either:

- Lines with negligible spectral width or;

- A tunable source with negligible spectral width and equal intensity as it is tuned over the ITF.

6. Slit width variation is negligible over the ITF centered at each particular wavelength.

Higher-order corrections will likely be needed in the future to account for the breakdown of some of these assumptions.

\section{Spectral Undersampling}

Consider a wavelength range extending from $\lambda_{\min }$ to $\lambda_{\max }$ that is fully or partly sampled by an array of detectors, spaced at wavelength increment $\delta \lambda$. Any continuous incoming signal spectrum extending over the wavelength range can be represented fully by expansion in spatial (i.e., wavelength) frequency in a Fourier series:

$$
S(\lambda)=a_{0}+\sum_{k=1}^{\infty}\left[a_{k} \cos k t+b_{k} \sin k t\right]=a_{0}+\sum_{k=1}^{\infty}\left[a_{k} \cos \left(\omega_{k}\left(\lambda-\lambda_{\min }\right)\right)+b_{k} \sin \left(\omega_{k}\left(\lambda-\lambda_{\min }\right)\right)\right]
$$

where

$t=2 \pi \frac{\lambda-\lambda_{\min }}{\lambda_{\max }-\lambda_{\min }} \equiv 2 \pi \frac{\lambda-\lambda_{\min }}{\Delta \lambda}$

and the spatial frequencies are:

$\omega_{k}=\frac{2 \pi k}{\Delta \lambda}$ 
Suppose that the signal is band-limited, that is, limited in content of spatial frequency components to a maximum spatial frequency $\omega_{\max }$. Then, by the sampling theorem, ${ }^{8,9}$ the information content of the spectrum is fully known if the spectrum is sampled over the full range $\lambda_{\min }$ to $\lambda_{\max }$ to twice this maximum spatial frequency, $2 \omega_{\max }$ (the Nyquist sampling frequency):

$$
\frac{\delta \lambda}{2 \pi}=\frac{1}{2 \omega_{\max }}
$$

In this case, the signal expansion includes only the terms necessary to measure spatial frequencies $\leq \omega_{\max }$ :

$$
S(\lambda)=a_{0}+\sum_{k=1}^{N}\left[a_{k} \cos k t+b_{k} \sin k t\right], \quad N=\frac{\Delta \lambda}{2 \delta \lambda}
$$

The spectrum is completely determined by this expansion. Its values at points other than the sampled points can be determined using the fact that the value at each sampled point $\lambda_{n}$ represents the intensity $c_{n}$ of a sampling function, sinc $\left[2 \pi\left(\lambda_{n}-\lambda\right) / \delta \lambda\right]$, centered at that point, ${ }^{8}$

$$
\operatorname{sinc}\left[2 \pi\left(\lambda_{n}-\lambda\right) / \delta \lambda\right]=\frac{\sin \left[2 \pi\left(\lambda_{n}-\lambda\right) / \delta \lambda\right]}{\left[2 \pi\left(\lambda_{n}-\lambda\right) / \delta \lambda\right]}
$$

plus the constant offset $a_{0}$ :

$$
c_{n}=S\left(\lambda_{n}\right)-a_{0}=\sum_{k=1}^{N}\left[a_{k} \cos k t_{n}+b_{k} \sin k t_{n}\right], \quad t_{n}=\frac{2 \pi\left(\lambda_{n}-\lambda_{\min }\right)}{\Delta \lambda}
$$

The sampling function is written here in this way to emphasize the pixel dependence and orientation of the following discussion. The sampling function presented here is actually an approximation to a fuller and more complex form, ${ }^{9,10}$ but the difference amounts to a completely negligible correction except within several sample points of the $\lambda_{\min }$ and $\lambda_{\max }$ endpoints.

If the signal is not band-limited to $\omega_{\max }$ but is still sampled to only $2 \omega_{\max }$, spatial frequencies greater than $\omega_{\max }$ are aliased into the band $0<\omega \leq \omega_{\max }$, with signal information for 
$\omega_{\max }<\omega \leq 2 \omega_{\max }$ appearing at $\omega_{\max }-\omega$, information for $2 \omega_{\max }<\omega \leq 3 \omega_{\max }$ appearing at $\omega-$ $2 \omega_{\max }$, etc. (Ref. 9, pp. 16-18). It will be demonstrated later that information aliased from the spatial frequency band $\omega_{\max }<\omega \leq 2 \omega_{\max }$ represents the most important sources of problems for GOME, and the main target of corrections. Problems from aliasing in the spectra become most evident when it becomes necessary to resample a spectrum in wavelength, for example when comparing an atmospheric radiance spectrum with a solar irradiance spectrum to determine atmospheric composition from molecular absorption lines, but the interference from aliasing is present in any case when the spectrum is undersampled (i.e., the spectrum is not fully Nyquist sampled). For example, synthetic spectra calculated during the fitting process to determine abundances of atmospheric gases would not normally include aliasing, while the measured spectra would.

A Nyquist-sampled spectrum $S(\lambda)$ is fully described by summing over the contributions from the $m$ individual sample points:

$$
S(\lambda)=c_{0}+\sum_{i=1}^{m} c_{i} \operatorname{sinc}\left[2 \pi\left(\lambda_{i}-\lambda\right) / \delta \lambda\right]
$$

It is now possible to investigate the case where the spectrum is not fully Nyquist sampled. Consider a spectrum input to the instrument $S_{\text {inp }}(\lambda)$. If the spectrum is completely known $a$ priori, it can be expanded as before in a Fourier series

$$
S_{i n p}(\lambda)=a_{0}+\sum_{k=1}^{\infty}\left[a_{k} \cos k t+b_{k} \sin k t\right]
$$

$S_{\text {inp }}$ can be separated into a Nyquist-sampled part, $S_{n y q}$, containing only spatial frequencies $\leq$ $\omega_{\max }$, and an undersampled part, $S_{\text {und }}$ :

$$
S_{n y q}(\lambda)=a_{0}+\sum_{k=1}^{N}\left[a_{k} \cos k t+b_{k} \sin k t\right]
$$


$S_{\text {und }}(\lambda)=\sum_{k=N+1}^{\infty}\left[a_{k} \cos k t+b_{k} \sin k t\right]$

The undersampled part of the spectrum is thus

$S_{\text {und }}(\lambda)=S_{i m p}(\lambda)-\sum_{i=1}^{m} c_{i} \operatorname{sinc}\left[2 \pi\left(\lambda_{i}-\lambda\right) / \delta \lambda\right]-c_{0}$

where $m$ runs over the sampled points.

\section{Slit Functions (Instrument Transfer Functions)}

An ITF serves as a low-pass filter to limit the spatial frequency content of the spectrum. Ideally, an ITF would limit the spectral information to frequencies $\leq \omega_{\max }$. The ITFs for satellite-based spectrometers are measured in one of three manners:

1. By using a reference line lamp (often a $\mathrm{PtNeCr}$ lamp "). This method has the advantage that lamp lines are much narrower than the ITF. The disadvantages are that lines are not always completely separated, that spectral coverage may be inadequate in some regions, and that a set of points (one per detector pixel) is mapped out, rather than a continuous ITF. This method was used for GOME and SCIAMACHY.

2. By using a tunable source consisting of a broadband light source and a monochromator. This method has the advantage of mapping out a continuous ITF, but the disadvantage of being a spectrally broader source than the PtNeCr line source. This method was used for OMI, where the full-width at half-maximum (FWHM) of the source was $\leq 0.1 \mathrm{OMI}$ detector pixel (M. Dobber and R. Dirksen, private communication, 2003). The residual effects from finite source width could, in principle, be reduced using the deconvolution techniques introduced below. 
3. By fitting flight spectral irradiance data to a high-resolution solar reference spectrum, ${ }^{3}$ where the fitting includes wavelength adjustment and simultaneous fitting to a parameterized ITF. ${ }^{2.12}$

An ITF, $\Gamma(\lambda)$, can be expanded to include Nyquist-sampled and undersampled portions, ignoring (for now) detector pixel binning:

$\Gamma(\lambda)=\gamma_{0}+\sum_{i=a}^{b} \gamma_{i} \operatorname{sinc}\left[2 \pi\left(\lambda_{i}-\lambda\right) / \delta \lambda\right]+\Gamma_{u n d}(\lambda)$.

The limits $a$ and $b$ are selected to include portions of the detector array where the slit function contributes significantly. As examples, we show the Gaussian GOME ITF we normally use for wavelength calibration purposes. A more complex, compound hypergeometric, ITF was determined during the instrument characterization. ${ }^{13}$ We find that the Gaussian spectrum provides better wavelength calibration for GOME spectra, and is used routinely in our data analyses. It also provides much better undersampling correction for GOME spectral fitting. We show an OMI ITF (M. Dobber and R. Dirksen, private communication, 2003) for this same wavelength region, and for the $\mathrm{NO}_{2}$ fitting region $(405-465 \mathrm{~nm}$; the ITF determined at $432 \mathrm{~nm}$ is used here). The OMI ITFs are selected for CCD row 150 , corresponding to a viewing azimuth angle of $29.5^{\circ}$, about half way from the nadir view to the extremity of the OMI swath. Additional types of ITFs for OMI may be considered once there is flight data for comparison. For all ITFs shown in this section, the complication of binning over the detector pixel response function is not yet included. This will be discussed in a later section.

Figure 1 shows the GOME Gaussian ITF, determined from ERS-2 orbit 81003031 (October 3, 1998), used here as a test orbit for determination of undersampling correction, appropriate to the fitting window used for BrO retrieval in GOME. ${ }^{2}$ The FWHM of the ITF is $0.160 \mathrm{~nm}$, and there are 1.4 samples per FWHM in this region of GOME spectra. The ITF is 
additionally decomposed into fully-sampled and undersampled components. Figure 2 shows this ITF with the hypothetical sampling to twice the GOME sampling frequency. Figure 3 shows the OMI ITF for this same wavelength region (FWHM $=0.421 \mathrm{~nm}, 2.8$ samples per FWHM), and its decomposition into fully-sampled and undersampled components. Figure 4 shows this TTF with the hypothetical sampling to twice the OMI sampling frequency. Figures 5 and 6 show the OMI ITFs and decompositions for the $\mathrm{NO}_{2}$ fitting wavelength range $(\mathrm{FWHM}=0.639 \mathrm{~nm}, 3.0$ samples per FWHM). Note the asymmetry in the measured OMI ITFs and their decompositions.

It is clear from all three examples that higher sampling by a factor of 2 greatly decreases the undersampled portion of the ITF, almost eliminating undersampling. Since for GOME this corresponds to 2.8 samples per FWHM, this demonstrates that information aliased from the spatial frequency band $\omega_{\max }<\omega \leq 2 \omega_{\max }$ represents the most important source of undersampling in this spectral region.

\section{Binning into Detector Pixels}

GOME and SCIAMACHY use Reticon-S RL-1024 SRU-type linear diode array detectors, which are designed to have the sensitivity profiles shown in Figure 7 for the spectral resolution and sampling of GOME. These detectors have 1024 photodiode elements spaced at $25 \mu \mathrm{m}$. The center $13 \mu \mathrm{m}$ of each element is n-doped, with the bulk of the material p-doped, to generate the response profile shown (the newer Reticon-L detectors are also spaced at $25 \mu \mathrm{m}$, but with $19 \mu \mathrm{m}$ $\mathrm{n}$-doped regions and $6 \mu \mathrm{m}$ interdiode gaps).

Characterization of the actual pixel responses must take into account the fact that the input light source (mostly Fraunhofer spectrum, convolved with the ILS) varies significantly across the detector pixel profile. The final response is the integral across the pixel profile of the convolution of the input spectrum with the ILS: 


$$
R_{p i x}(\lambda)=\int_{\text {prosile }} S\left(\lambda^{\prime}\right) \otimes \Gamma\left(\lambda-\lambda^{\prime}\right) d \lambda^{\prime}
$$

where $R_{p i x}$ is the pixel response, $S$ is the input spectrum, $\Gamma$ is the ILS, and $\otimes$ denotes convolution. In order to calculate the response, it is first necessary to obtain the ILS by deconvolution, since the measured ITF is the convolution of the ILS with the pixel response profile. This is accomplished using the Jansson method, as described in Ref. 14, for GOME, and a nonlinear least squares fitting to a parameterized profile shape for OMI. The result for the GOME slit function is shown in Figure 8 . The deconvolved slit is the best match to the ILS that could be obtained in the iterative deconvolution process. The difference between the initial and reconvolved slit is an indicator of the goodness-of-fit for this procedure. The response functions for the OMI CCD detectors are Gaussian with a full-width at half maximum of $25 \mu \mathrm{m}$ and separated by $22.5 \mu \mathrm{m}$ (M. Dobber and R. Dirksen, private communication, 2003). Figures 9 and 10 show these along with the decomposition to determine the ILS for the $\mathrm{BrO}$ and $\mathrm{NO}_{2}$ fitting regions of OMI.

\section{Undersampling Correction}

\subsection{Wavelength calibration issues}

Satellite radiances and irradiances can be calibrated in wavelength with high absolute accuracy (typically $\leq 0.0004 \mathrm{~nm}$ for the GOME BrO fitting region) using cross-correlation to the solar

spectrum of Ref. 3, with simultaneous fitting of the ITF. ${ }^{2,12}$ Ref. 2 suggested that the apparent wavelength shift between GOME radiances and irradiances was due to instrumental effects, influencing the way the detectors are illuminated in the different measuring geometries. Ref. 4 stated that it is due to the Doppler effect, since irradiances are obtained during the portion of the 
orbit when the satellite is moving toward the sun. For ERS-2 orbit 81003031, the maximum velocity toward the sun is $7.46 \mathrm{~km} \mathrm{~s}^{-1}$, while the average wavelength shift (irradiance - radiance) for the $\mathrm{BrO}$ fitting window is equivalent to $7.865 \pm 0.025 \mathrm{~km} \mathrm{~s}^{-1}(0.00923 \pm 0.00003 \mathrm{~nm})$. The satellite Doppler shift contributes $6.89 \mathrm{~km} \mathrm{~s}^{-1}(0.0081 \mathrm{~nm})$, rather than the full $7.46 \mathrm{~km} \mathrm{~s}^{-1}$, given the GOME solar measurement procedure. ${ }^{4}$ There is an additional component of $0.50 \mathrm{~km} \mathrm{~s}^{-1}$ at this season from the ellipticity of the Earth's orbit, for a total Doppler shift of $7.39 \mathrm{~km} \mathrm{~s}^{-1}$, leaving a significant instrument and/or spectral component (see the discussion in Ref. 15 for possible spectral "tilt" contributions). For the other spectrometers considered here, the relative contributions are not yet determined. For OMI in particular, since its undersampling is calculated here, a relative shift equal to the full Doppler shift is assumed; this will be modified when flight data become available.

\subsection{Undersampling calculations}

Calculation of the undersampling correction for each case is accomplished by convolving the high spectral resolution $(0.01 \mathrm{~nm})$ solar reference spectrum of Ref. 3 with the ITF determined for the instrument and wavelength region, and differencing fully-sampled and undersampled representations of this convolved solar spectrum at the sampling grid of the satellite radiances:

1. Convolve the high resolution solar reference spectrum $E_{r e f}$ with the satellite instrument ITF to create a lower spectral resolution, but highly oversampled, solar reference spectrum, $E_{\text {over }}$ :

$$
E_{\text {over }}(\lambda)=\int_{\text {profile }} E_{\text {ref }}\left(\lambda^{\prime}\right) \otimes \operatorname{ITF}\left(\lambda-\lambda^{\prime}\right) d \lambda^{\prime}
$$

The portion of $E_{\text {ref }}$ used in fitting BrO for GOME is shown in Figure 11, top panel; $E_{\text {over }}$ for this spectral region is shown in Figure 11, middle panel. 
2. Determine from direct cross-correlation (for GOME) or estimate (for OMI) the wavelength grids for irradiance and radiance spectra $g_{i r}$ and $g_{\text {rad }}$.

3. Sample $E_{\text {over }}$ at the wavelength grid $g_{i r r}$ to give $E_{i r r}$, and at the grid $g_{\text {rad }}$, to give $E_{\text {rad, }}$, using cubic spline interpolation ${ }^{16}$ to determine values at each exact grid point. These are now undersampled representations of the solar reference spectrum, although each is correct at the points on its sampling grid. $E_{i r r}$ is shown in Figure 11, bottom panel; $E_{\text {rad }}$ is virtually indistinguishable when over-plotted.

4. Resample $E_{i r}$ to the wavelength grid $g_{r a d}$, using cubic spline interpolation, giving $E_{r a d}^{\prime}$.

5. The undersampling correction $C_{u}$, in optical thickness units, is the difference between $E_{r a d}$ and $E_{r a d}^{*}$, normalized to the average of $E_{r a d}$ over the fitting window:

$$
C_{u}(\lambda)=\frac{E_{r a d}(\lambda)-E_{r a d}^{\prime}(\lambda)}{\bar{E}_{r a d}} .
$$

$C_{u}$ corresponds to differencing fully-sampled and undersampled representations of the convolved solar spectrum since only $E_{\text {irr }}$ is resampled, thus inducing undersampling error on the radiance wavelength grid $g_{r a d}$. This undersampling correction assumes that, to first order, the radiance spectrum consists mostly of back scattered Fraunhofer structure. Higher-order corrections could be made to account for atmospheric absorption and the Ring effect. Figure 12 shows the residuals from fitting for $\mathrm{BrO}$ (without the use of an undersampling spectrum as a basis function) in ERS-2 orbit 81003031 for a measurement pixel midway through the orbit (pixel 800) and as an orbit average (top panel); the bottom panel shows the undersampling $C_{u}$ calculated here for the average relative wavelength shift between irradiance and radiance fitted for this orbit $(0.0092$ $\mathrm{nm})$. The calculated undersampling correction accounts for more than $90 \%$ of the fitting residuals. Figure 13, top panel, shows the undersampling calculated for the OMI baseline $\mathrm{BrO}$ fitting window, assuming a relative radiance-irradiance shift corresponding to the full Doppler 
shift for the Aura orbit $\left(7.5 \mathrm{~km} \mathrm{~s}^{-1}\right)$. The bottom panel shows the absorption for a typical BrO slant column density of $1 \times 10^{14} \mathrm{~cm}^{-2}$. The units (optical thickness and transmission) are equivalent in scale for these small interferences and absorptions. The undersampling correction is roughly an order of magnitude smaller than for GOME, but is still larger than the BrO absorption and needs to be carefully included in the fitting of the satellite data. Figure 14, top panel, shows the undersampling calculated for the $\mathrm{OMI}$ baseline $\mathrm{NO}_{2}$ fitting window, assuming a relative radiance-irradiance shift corresponding to the full Doppler shift for the Aura orbit. The bottom panel shows the absorption for a typical $\mathrm{NO}_{2}$ North American summer slant column density of $1.25 \times 10^{16} \mathrm{~cm}^{-2}$. The contribution is less severe but, at ca. $20 \%$ of the nominal $\mathrm{NO}_{2}$ signal, it is still by no means negligible. Undersampling correction will very likely need to be included in order to derive meaningful tropospheric $\mathrm{NO}_{2}$ abundances from OMI measurements. Under heavily polluted conditions over North America in summertime, for example, the tropospheric contribution to the $\mathrm{NO}_{2}$ slant column usually does not exceed $40 \%{ }^{6}$ Effective monitoring of moderate pollution requires correction to substantially better than that level.

An attempt was made to improve the undersampling correction for GOME by calculation of the undersampling spectrum for the deconvolved ILS, followed by convolution of the resulting spectrum with the Reticon response function. The result of this procedure was expected to be an improved undersampling correction which would correspond even more closely to the GOME fitting residual, as shown in Figure 12, top panel. The actual result was substantially worse. The magnitude was approximately correct, but the spectral details were not. We think that the reason is that the actual detector response function deviates considerably from the trapezoidal shape shown in Figure 7. For the present, at least, the best undersampling corrections continue to be those determined using the entire ITF. These are shown in Figures 12, 13, and 14. When OMI 
flight data become available, this procedure will be attempted to see whether it provides improved undersampling correction for this case.

\section{Discussion and Conclusions}

We provide a method that may be used to simply and reliably estimate the degree of undersampling an instrument configuration will have. For instruments where the ITF may be approximated as Gaussian (GOME), we show that sampling at 2.8 pixels/FWHM will almost completely eliminate undersampling. For more complex ITFs (OMI), significant undersampling can exist at 3.0 pixels/FWHM.

Undersampling for ground-based, zenith-sky spectrometers has been discussed in Ref. 17 in the context of measuring $\mathrm{O}_{3}, \mathrm{NO}_{2}$, and $\mathrm{NO}_{3}$. On the basis of numerical experiments, it is recommend to use sampling ratios between 4.5 and 6.5 pixels/FWHM, for Gaussian ITFs, to avoid undersampling. This is consistent with our finding that for $\mathrm{OMI} \mathrm{NO}_{2}$ measurements undersampling will be significant, and will highly impact tropospheric $\mathrm{NO}_{2}$ measurements, at 3.0 pixels/FWHM, but that at 6.0 pixels/FWHM it becomes negligible. The improvement in undersampling of OMI over that of GOME is due to the higher sampling rates (2.8 and 3.0 samples per FWHM for the OMI UV and visible examples versus 1.4 for the GOME UV example). Asymmetric ITFs such as those of OMI and perhaps other imaging spectrometers may require higher sampling ratios than symmetric ITFs.

The previously developed undersampling correction ${ }^{2,4}$ is now commonly used in GOME scientific analyses and has been implemented operationally for SCIAMACHY. It has been demonstrated here that correction will be required for $\mathrm{OMI} \mathrm{BrO}$ and tropospheric $\mathrm{NO}_{2}$ measurements, at least. Complete implementation will not be possible until the ITFs and 
irradiance-radiance wavelength shifts are characterized in flight versus $C C D$ row. A similar conclusion almost certainly applies to the OMPS instruments.

Further refinement of the undersampling correction for OMI, to include full averaging of the solar spectrum over the ILS and convolution with the pixel response is currently in progress, to be ready for application to flight spectra. For GOME, this further refinement has been shown not to be an improvement, likely due to incorrect characterization of the Reticon response function. Convolution with the ITF followed by the sampling procedure described above in Undersampling calculations provides very accurate correction.

Higher order corrections for instrumental effects, such as uneven sampling in wavelength space, and spectroscopic effects, such as atmospheric absorption and the Ring effect, may eventually be developed if analysis of flight data indicates that they are warranted.

\section{Acknowledgements}

This research was funded by the National Aeronautical and Space Administration and the Smithsonian Institution. We thank Marcel Dobber and Ruud Dirksen of the KNMI and Ruud Hoogeveen of the SRON for help with information on GOME and OMI detector responses and

ITF determinations. It is always a pleasure to acknowledge the European Space Agency and the German Aerospace Center for their ongoing cooperation in GOME and SCIAMACHY.

\section{References}

1. European Space Agency, "The GOME Users Manual," ed. F. Bednarz, European Space Agency Publication SP-1182, ESA Publications Division, ESTEC, Noordwijk, The Netherlands, ISBN-92-9092-327-x (1995). 
2. K. Chance, Analysis of BrO measurements from the Global Ozone Monitoring Experiment. Geophys. Res. Lett. 25, 3335-3338 (1998).

3. K.V. Chance and R.J.D. Spurr, Ring effect studies: Rayleigh scattering, including molecular parameters for rotational Raman scattering, and the Fraunhofer spectrum, Appl. Opt. 36, 5224-5230 (1997).

4. S. Slijkhuis, A. von Bargen, W. Thomas, and K. Chance, Calculation of undersampling correction spectra for DOAS spectral fitting, Proc. ESAMS 99 - European Symposium on Atmospheric Measurements from Space, 563-569 (1999).

5. K. Chance, P.I. Palmer, R.J.D. Spurr, R.V. Martin, T.P. Kurosu, and D.J. Jacob, Satellite observations of formaldehyde over North America from GOME, Geophys. Res. Lett. 27, $3461-3464$ (2000).

6. R.V. Martin, K. Chance, D.J. Jacob, T.P. Kurosu, R.J.D. Spurr, E. Bucsela, J.F. Gleason, P.I. Palmer, I. Bey, A.M. Fiore, Q. Li, and R.M. Yantosca, An improved retrieval of tropospheric nitrogen dioxide from GOME, J. Geophys. Res. 107, 4437, doi:10.1029/2001JD0010127 (2002).

7. NASA, “OMI Algorithm Theoretical Basis Document Volume 4: Trace Gas Algorithms," ed. K. Chance (2002). Available at http://eospso.gsfc.nasa.gov/eos_homepage/for_scientists/atbd/

8. S. Goldman, "Information Theory," Prentice-Hall, NY (1953).

9. R.W. Hamming, “Digital Filters,” Prentice-Hall, Englewood Cliffs, NJ, ISBN 0-13-212571-4 (1977).

10. J.C. Brasunas, Jr., "Far-Infrared Spectroscopy of Gaseous Nebulae," Ph.D. Thesis, Department of Physics, Harvard University (1981). 
11. J.E. Murray, "Atlas of the Spectrum of a Platinum/Chromium/Neon Hollow-Cathode Reference Lamp in the Region 240-790 nm," ESA Report (1994).

12. C. Caspar and K. Chance, GOME wavelength calibration using solar and atmospheric spectra, Proc. $3^{\text {rd }}$ ERS Symposium on Space at the Service of our Environment, ed. T.-D. Guyenne and D. Danesy, European Space Agency publication SP-414, ISBN 92-9092-656-2 (1997).

13. R. Spurr and W. Thomas, GOME software databases for level 1 to 2 processing, ER-TN-IFEGO-0018, Iss./Rev.3/A (2002). Available at http://earth.esa.int/services/esa_doc/doc_gom.html

14. W.E. Blass and G.W. Halsey, "Deconvolution of Absorption Spectra," Academic Press, NY, ISBN 0-12-104650-8 (1981).

15. C.E. Sioris, C.S. Haley, C.A. McLinden, C. von Savigny, I.C. McDade, W.F.J. Evans, J.C. McConnell, N.D. Lloyd, E.J. Llewellyn, D. Murtagh, U. Frisk, T.P. Kurosu, K.V. Chance, K. Pfeilsticker, H. Bösch, and F. Weidner, Stratospheric profiles of nitrogen dioxide observed by Optical Spectrograph and Infrared Imager System on the Odin satellite, J. Geophys. Res. 108, No. D7, 4215, doi:10.1029/2002JD002672 (2003).

16. W.H. Press, P.B. Flannery, S.A. Teukolsky, and W.A. Vetterling, "Numerical Recipes," Cambridge University Press, ISBN 0-521-30811-9 (1986).

17. H.K. Roscoe, D.J. Fish, and R.L. Jones, Interpolation errors in UV-visible spectroscopy for stratospheric sensing: Implications for sensitivity, spectral resolution, and spectral range, Appl. Opt. 35, 427-432 (1996). 


\section{Figure Captions}

Fig. 1 GOME Gaussian instrument transfer function in the spectral region used for bromine monoxide determinations, and its decomposition into Nyquist-sampled and undersampled components.

Fig. 2 GOME Gaussian instrument transfer function and the Nyquist-sampled and undersampled portions for the hypothetical case where the slit function is sampled to twice the GOME spatial frequency.

Fig. $3 \mathrm{OMl}$ instrument transfer function for the BrO fitting region and the Nyquist-sampled and undersampled portions.

Fig. $4 \mathrm{OMI}$ instrument transfer function for the BrO fitting region and the Nyquist-sampled and undersampled portions for the hypothetical case where the slit function is sampled to twice the OMI spatial frequency.

Fig. $5 \mathrm{OMI}$ instrument transfer function for the $\mathrm{NO}_{2}$ fitting region and the Nyquist-sampled and undersampled portions.

Fig. $6 \mathrm{OMI}$ instrument transfer function for the $\mathrm{NO}_{2}$ fitting region and the Nyquist-sampled and undersampled portions for the hypothetical case where the slit function is sampled to twice the OMI spatial frequency.

Fig. 7 Response of three Reticon-S detector pixels as a function of location in GOME detector channel 2.

Fig. 8 Deconvolution of the GOME ITF from the pixel response function to determine the ILS in the BrO fitting region.

Fig. 9 Deconvolution of the OMI ITF from the pixel response function to determine the ILS in the BrO fitting region. 
Fig. 10 Deconvolution of the OMI ITF from the pixel response function to determine the ILS in the $\mathrm{NO}_{2}$ fitting region.

Fig. 11 The portion of the high resolution solar reference spectrum $E_{\text {ref }}$ used in fitting BrO for GOME (top); $E_{\text {ref }}$ convolved with the GOME ITF to create the lower spectral resolution, but highly oversampled, solar reference spectrum, $E_{\text {over }}$ (middle); $E_{\text {over }}$ sampled at the wavelength grid of the GOME irradiance $\left(E_{i r r}\right)$ (bottom). $E_{i r r}$ and $E_{\text {rad }}$ (not plotted here; see text) are undersampled representations of the solar reference spectrum.

Fig. 12 Residuals from fitting GOME spectra for BrO in ERS-2 orbit 81003031 , for a single spectrum and as an average for all spectra in the orbit (top); Synthetic undersampling, $C_{u}$, calculated here for the relative wavelength shift between the GOME irradiance and the GOME radiance (bottom).

Fig. 13 Synthetic $\mathrm{OMI}$ undersampling, $C_{u}$, in the $\mathrm{BrO}$ fitting region, calculated here for a relative wavelength shift of the irradiance and radiance corresponding to $7.5 \mathrm{~km} \mathrm{~s}^{-1}$ (top); absorption for a typical BrO slant column density of $1 \times 10^{14} \mathrm{~cm}^{-2}$ (bottom).

Fig. 14 Synthetic OMI undersampling, $C_{u}$, in the $\mathrm{NO}_{2}$ fitting region, calculated here for a relative wavelength shift of the irradiance and radiance corresponding to $7.5 \mathrm{~km} \mathrm{~s}^{-1}$ (top); absorption for a typical $\mathrm{NO}_{2}$ slant column density of $1.25 \times 10^{16} \mathrm{~cm}^{-2}$ (bottom). 


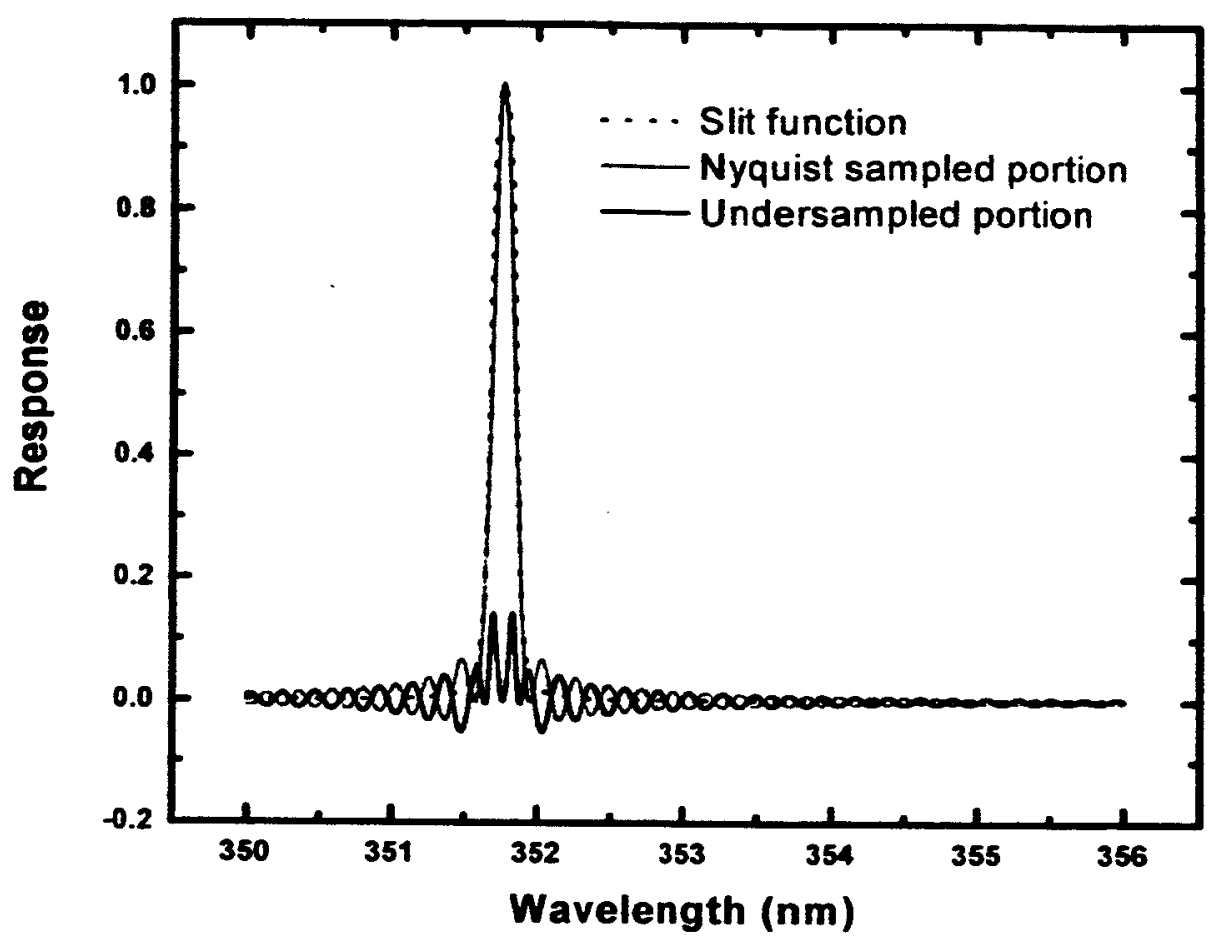




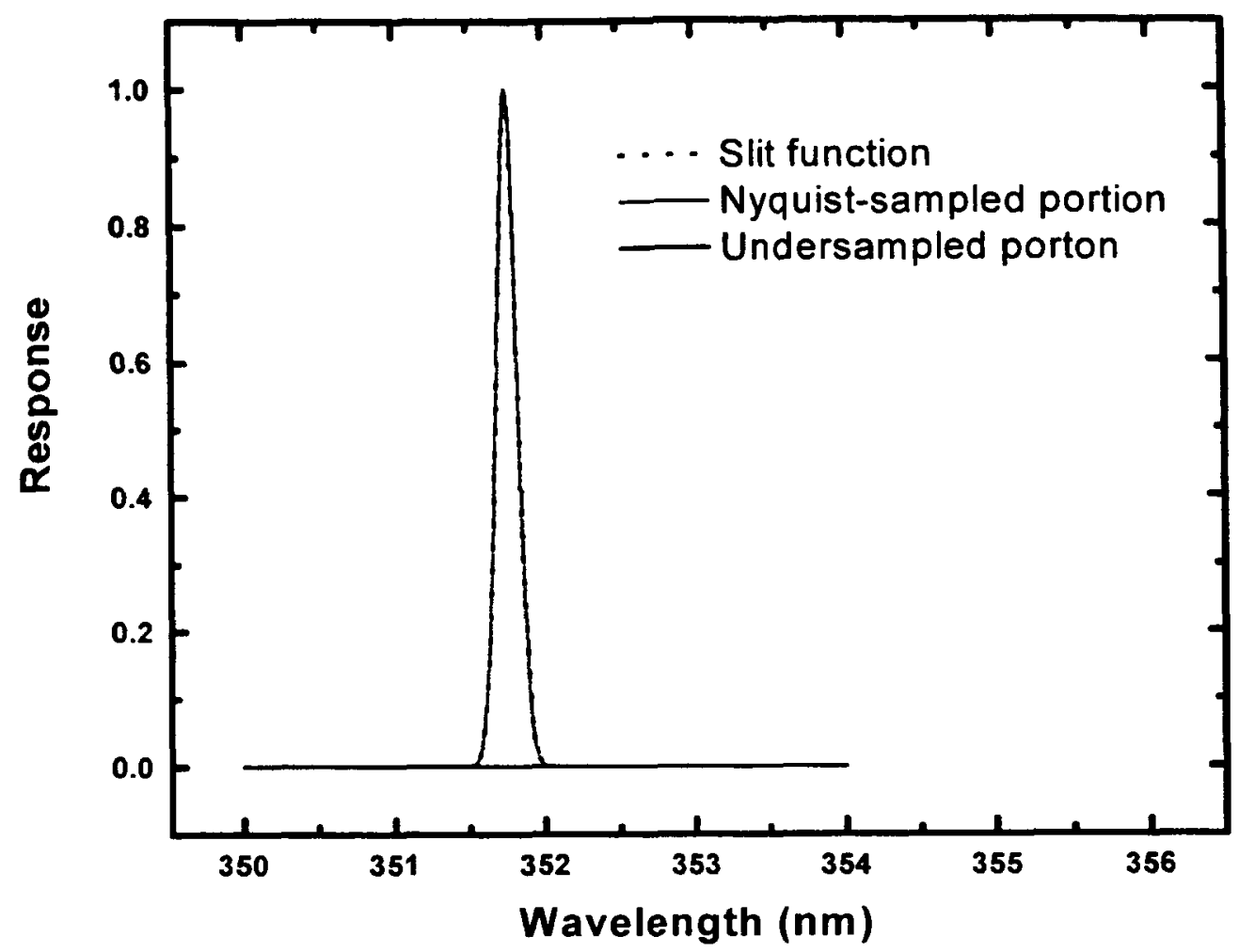




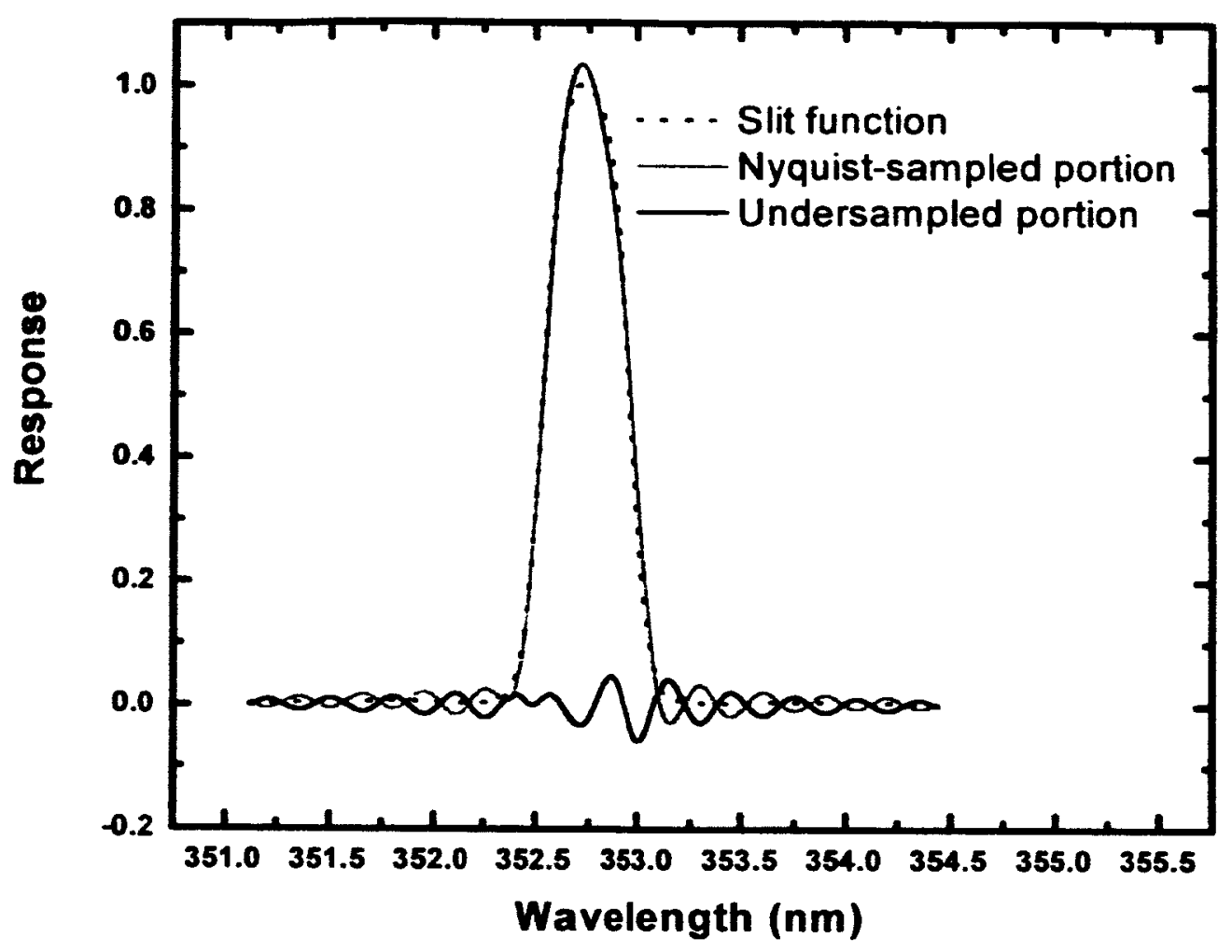




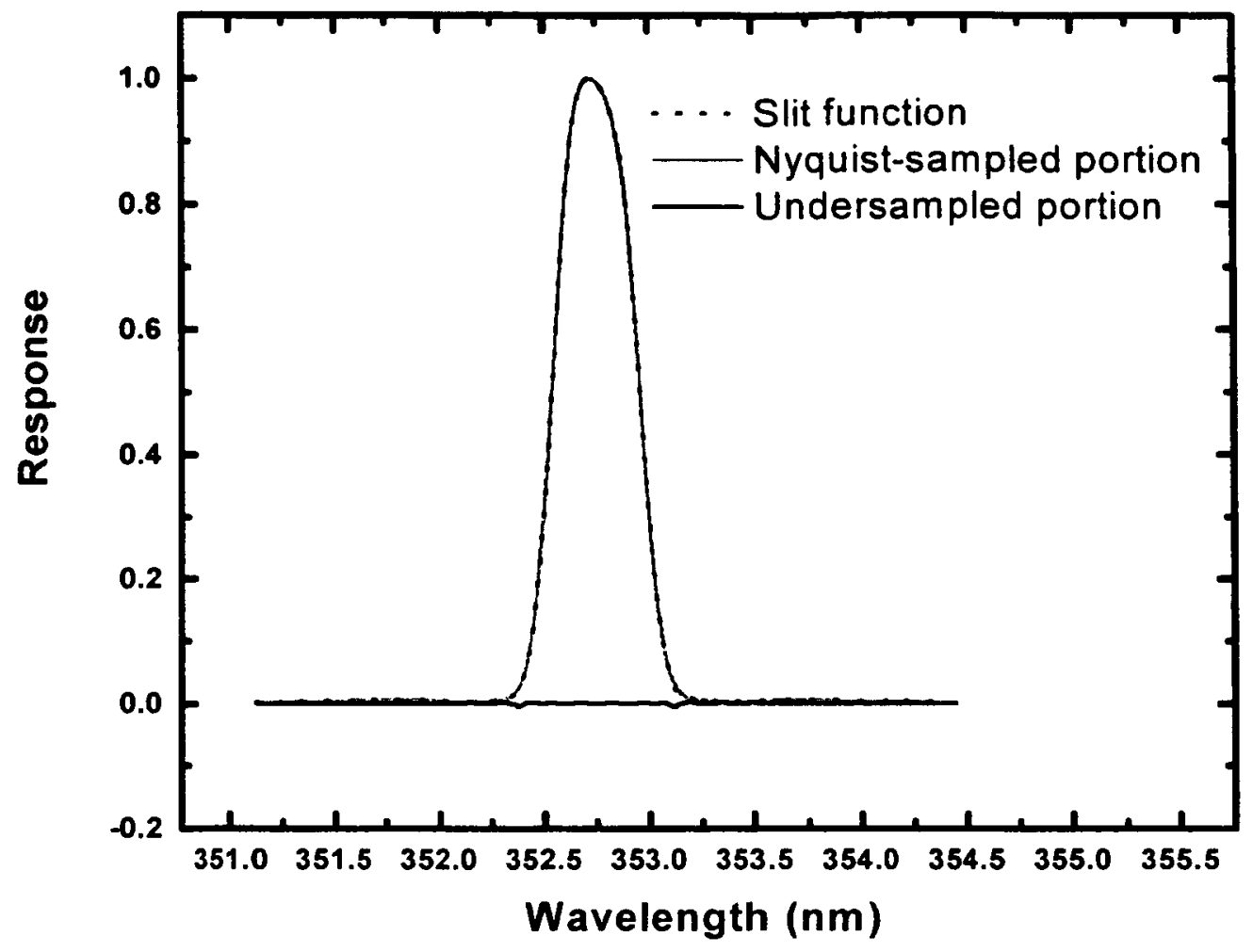




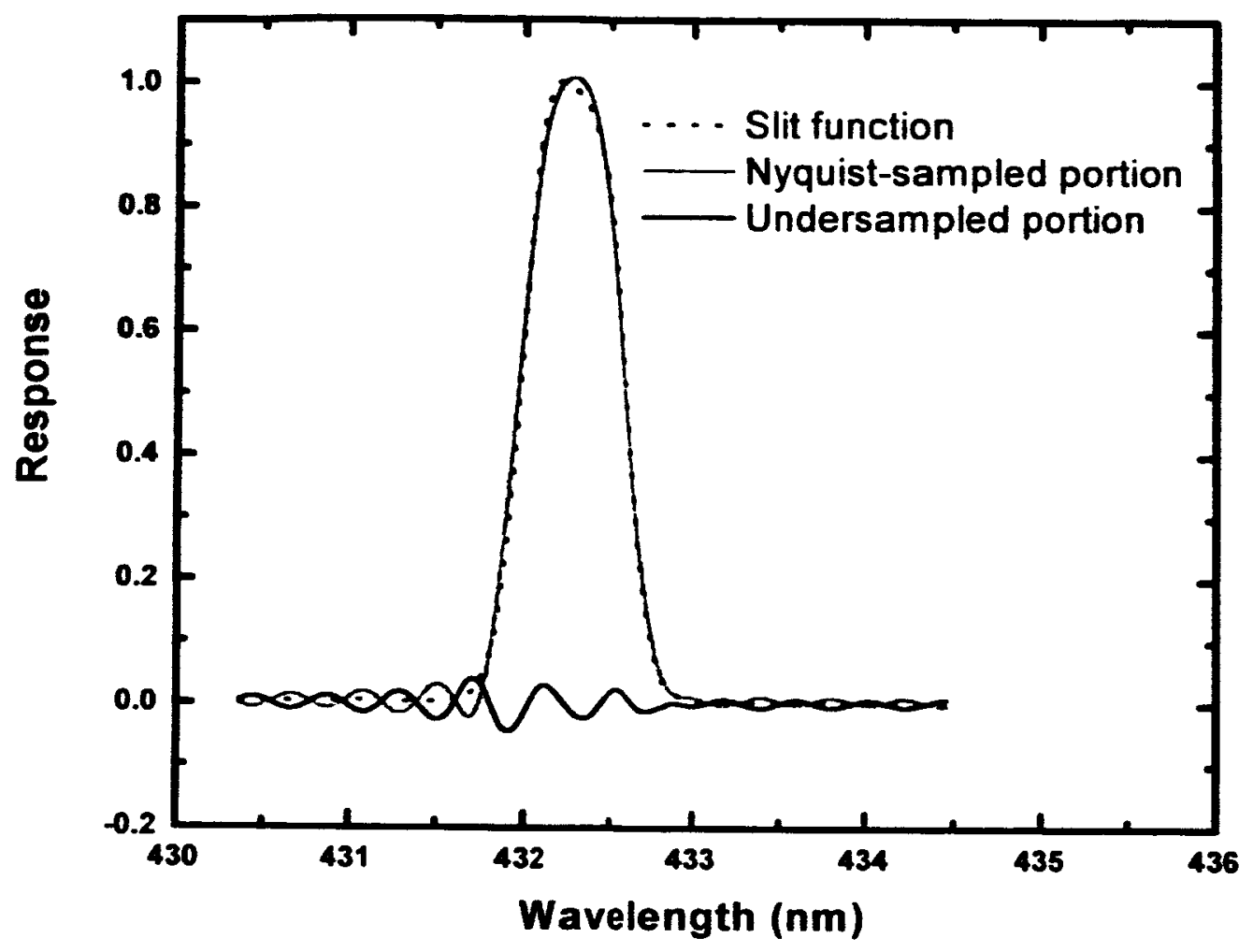




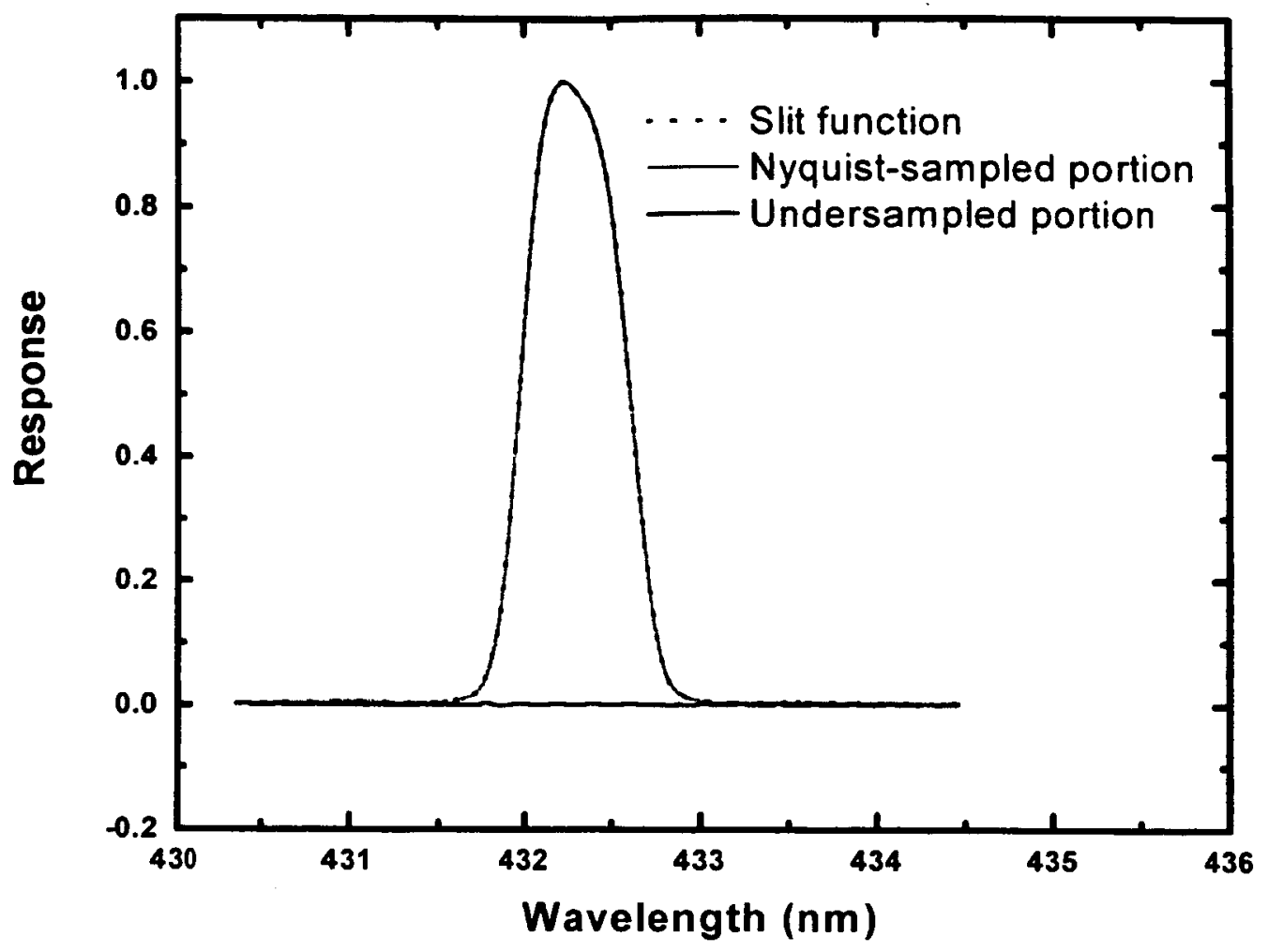



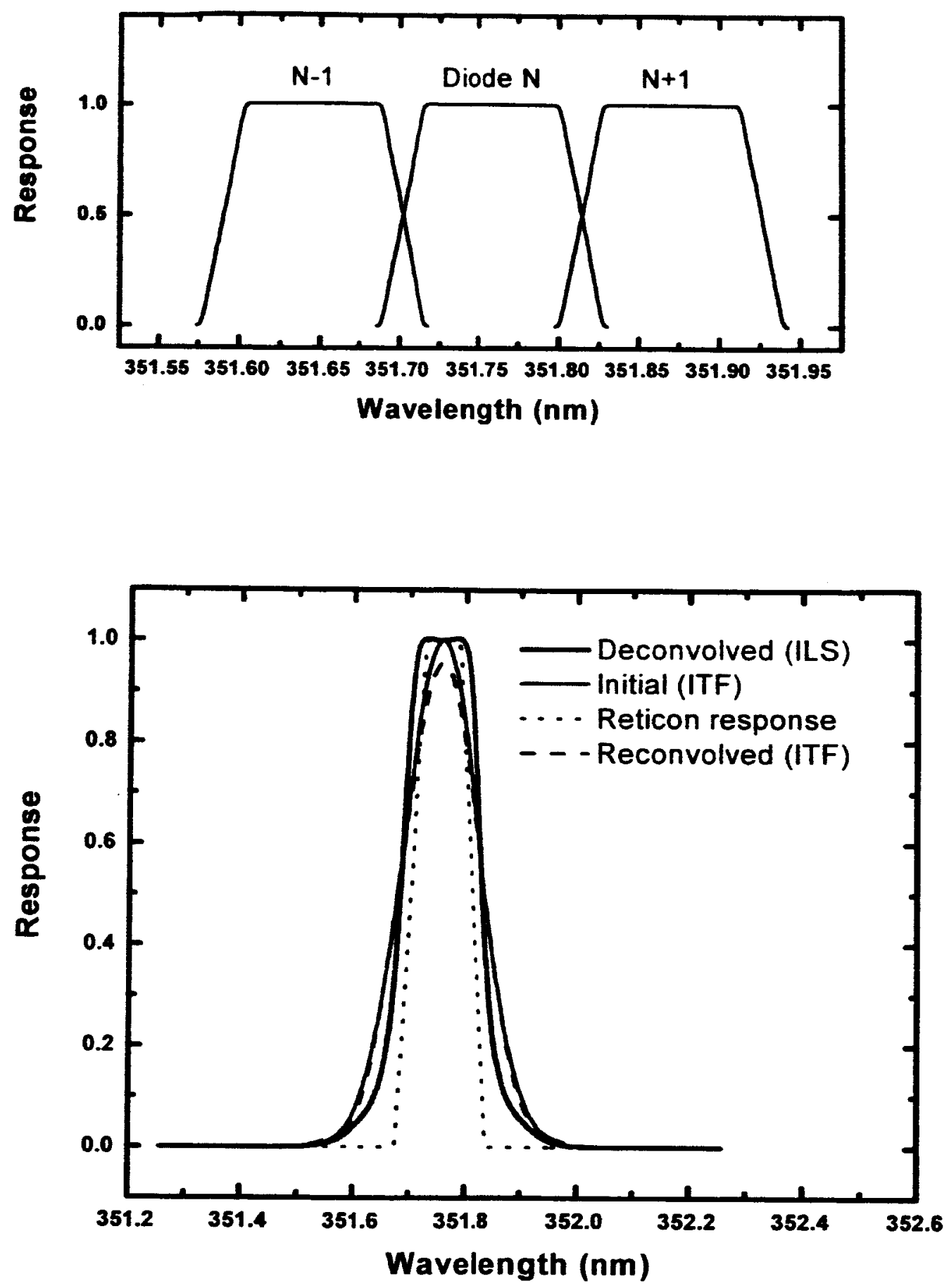


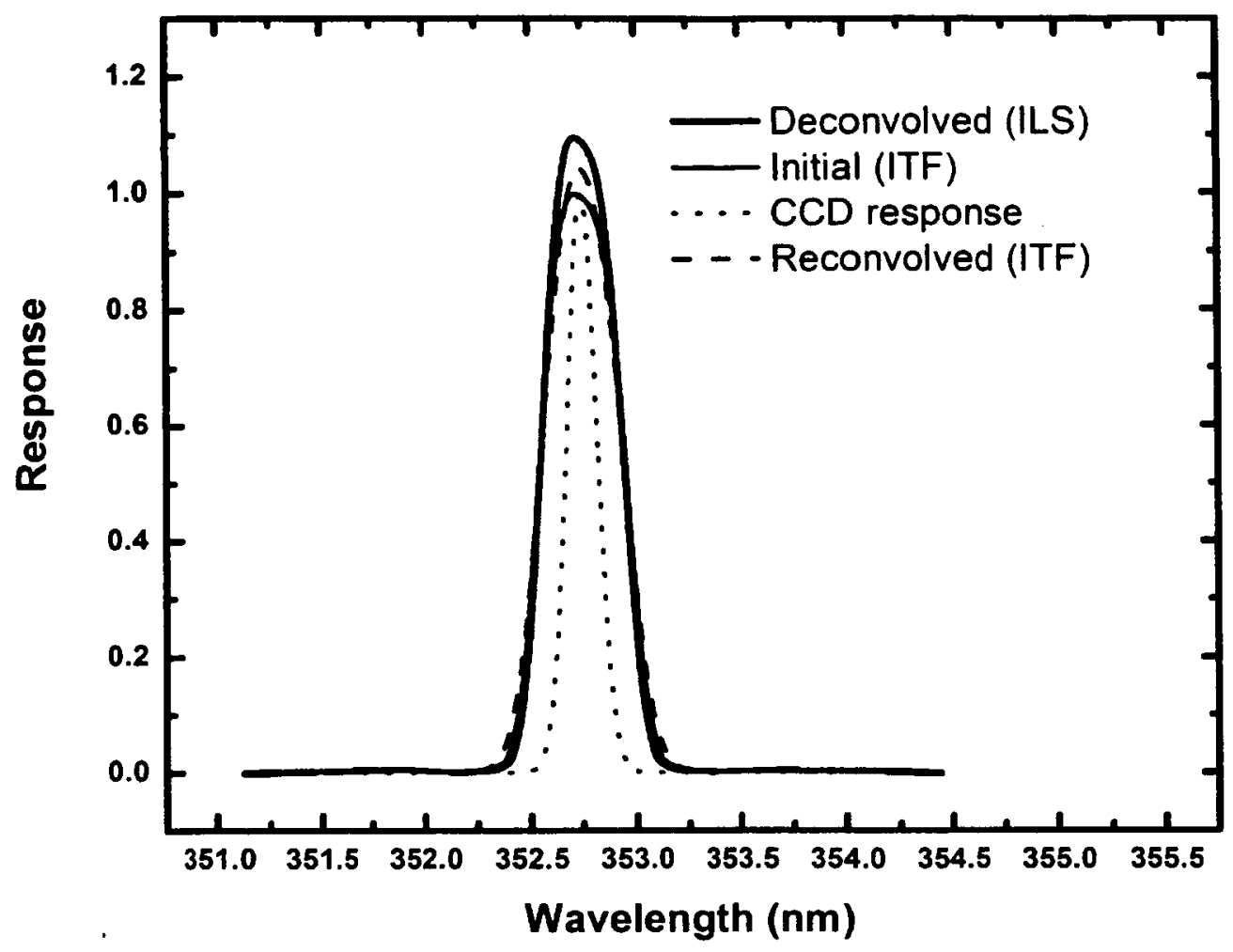




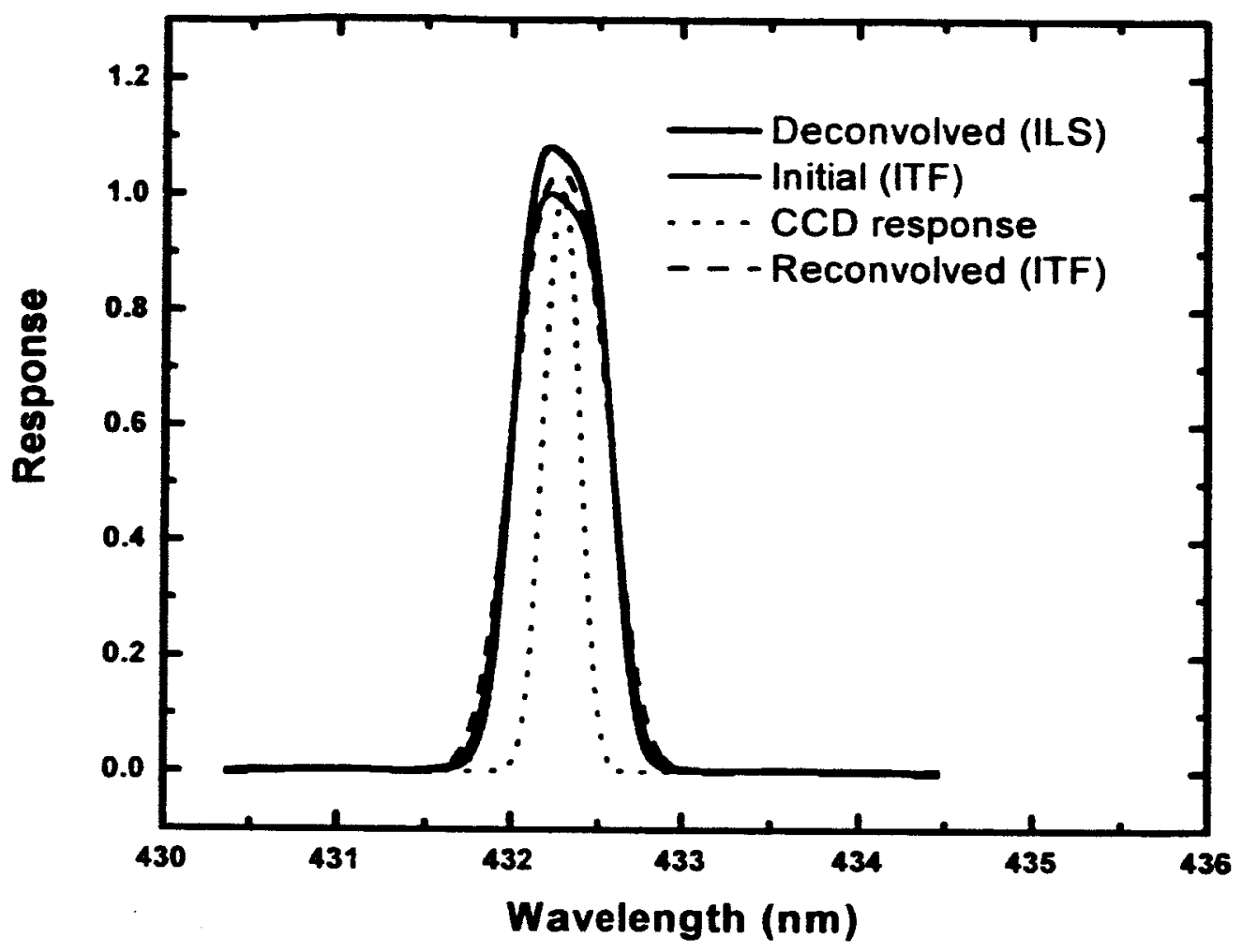



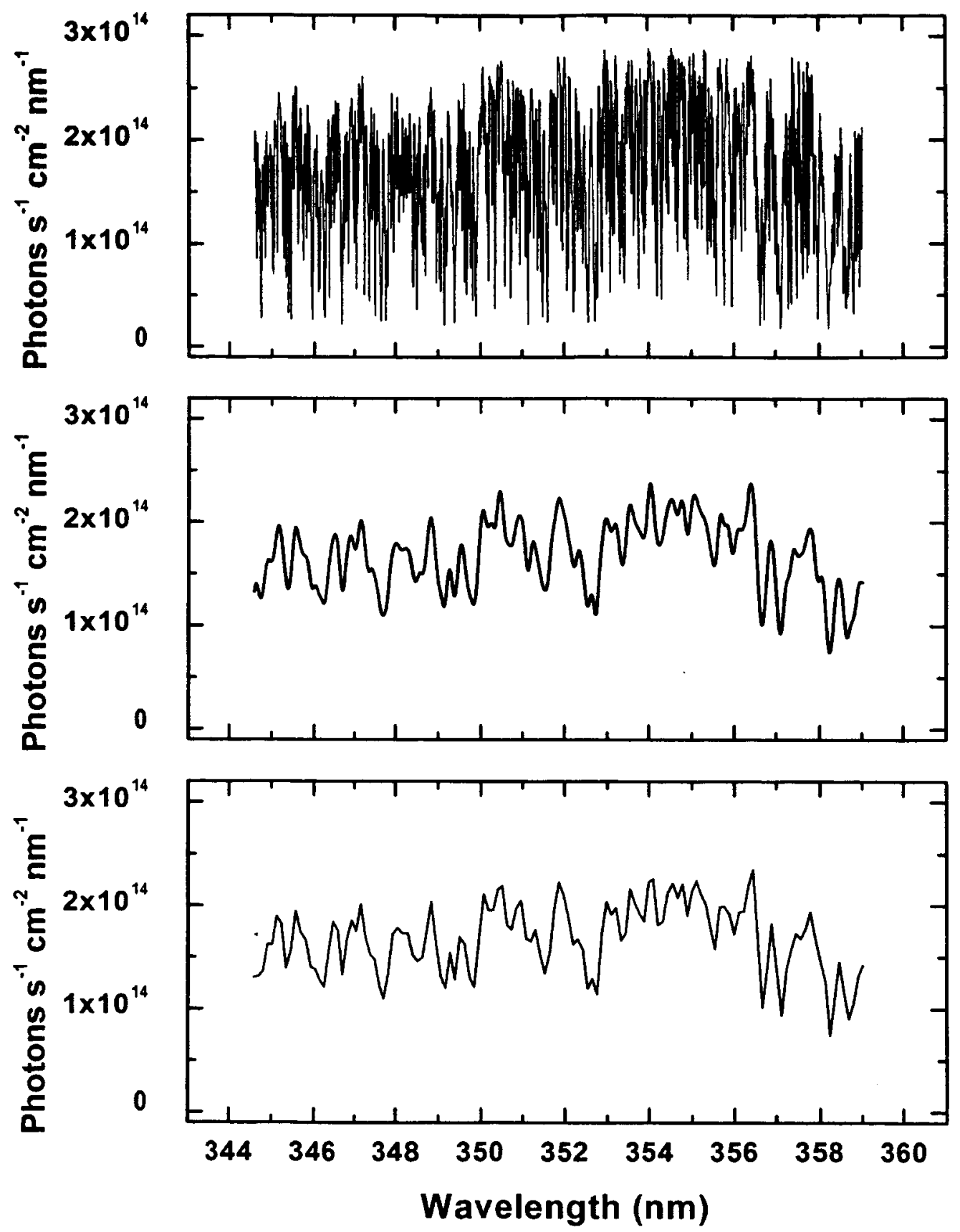

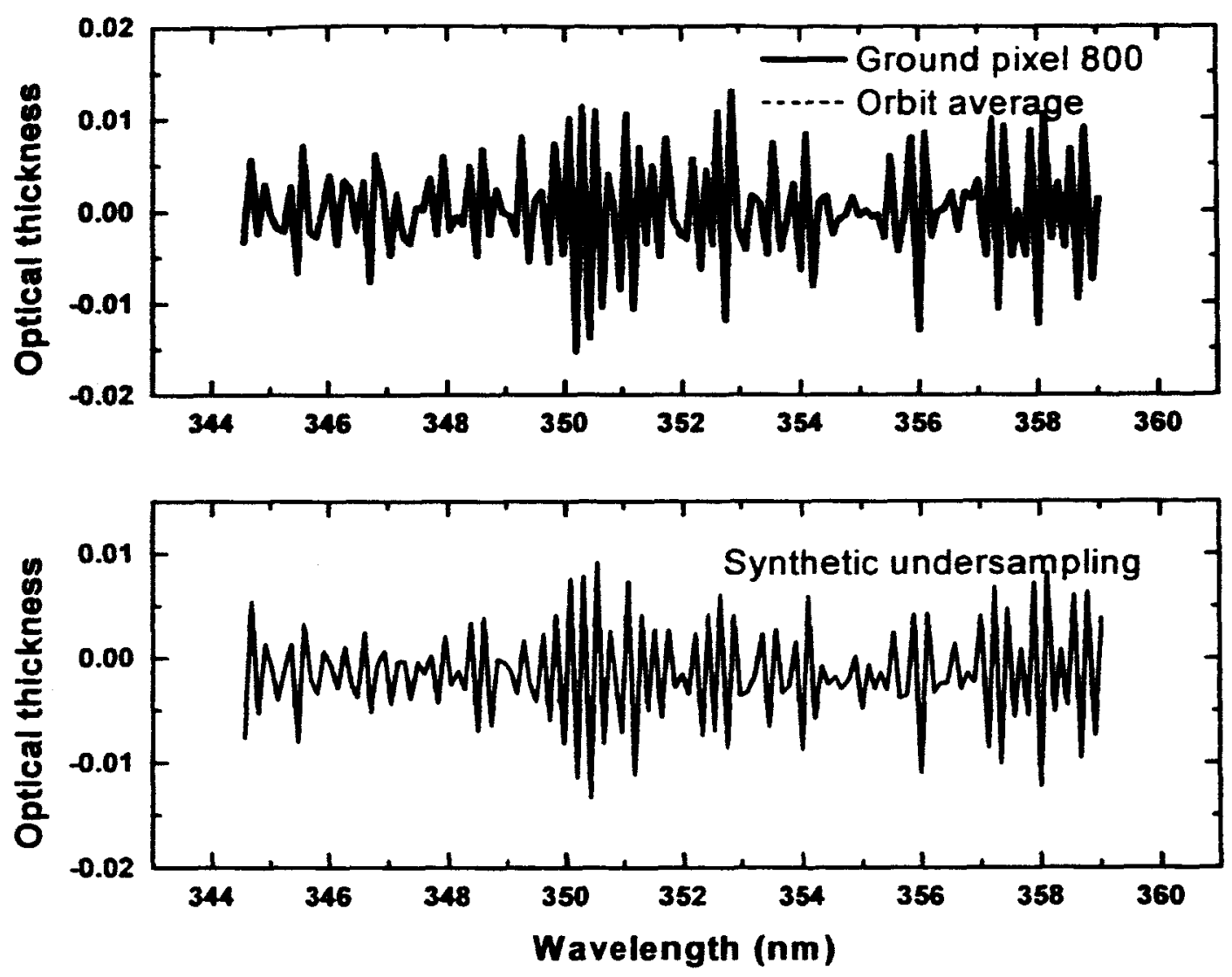

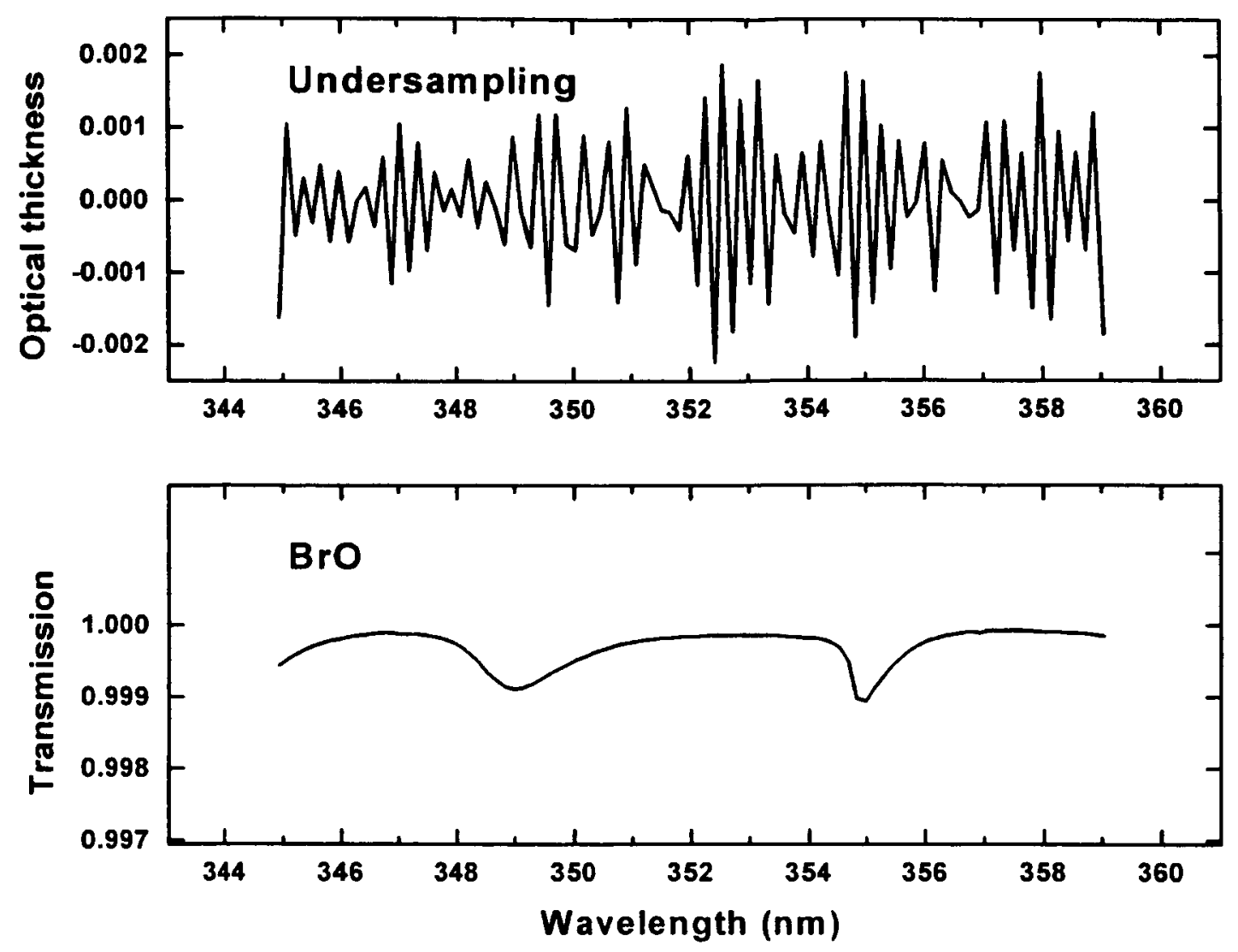

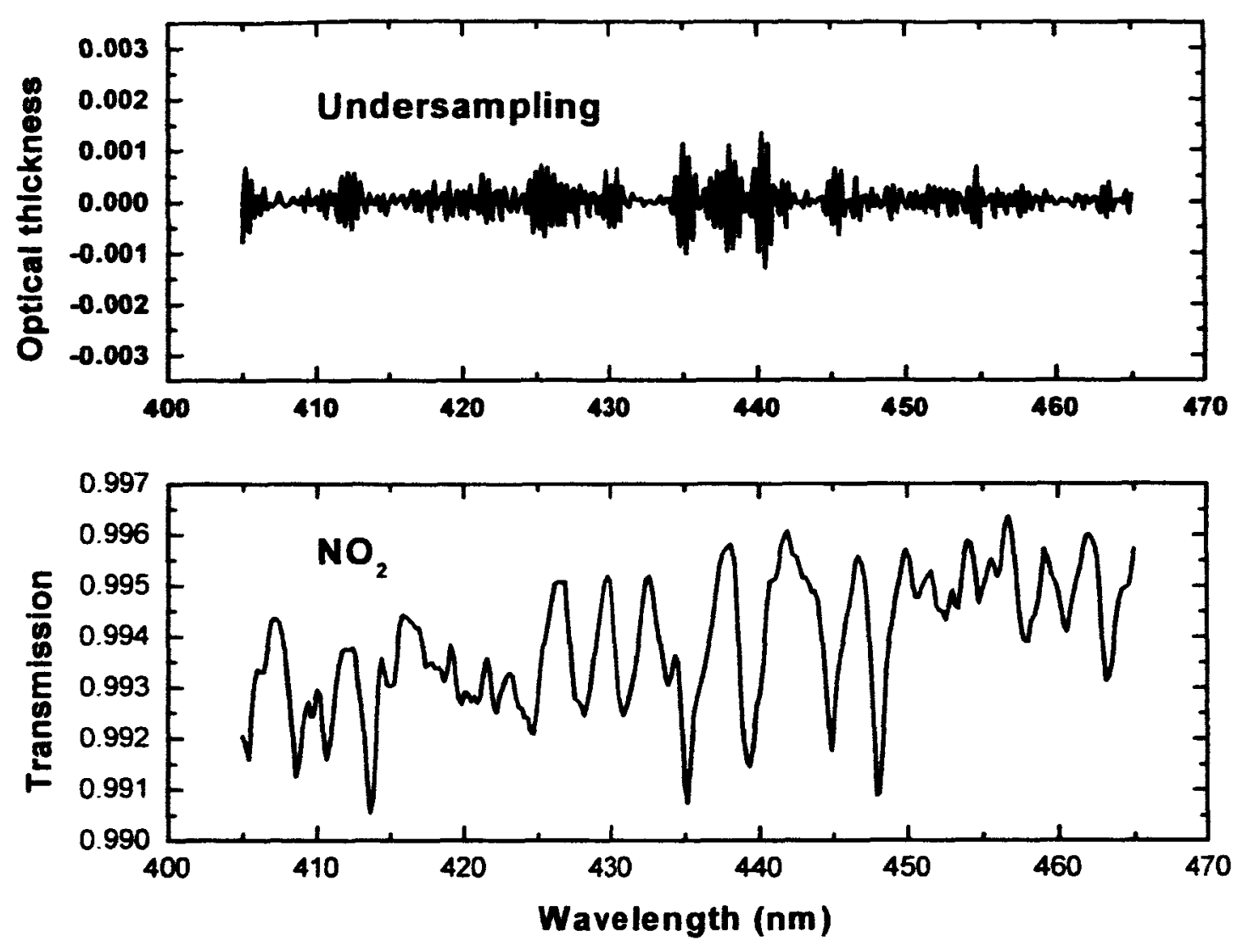